\title{
A Distillation Approach to Phase Equilibrium Measurements of Multicomponent Fluid Mixtures
}

Supporting Information

Updated June 6, 2019

Comparison to previous ADC measurements. Before the initiation of a comprehensive study of $\mathrm{C} 10 / \mathrm{C} 14$ vapor-liquid equilibrium using ADCR, the modified apparatus was tested by distilling a 50/50 (mol/mol) mixture to ensure that the distillation curve measured by the classical ADC in Bruno 2006 was replicable. ${ }^{1}$ The curves, which were measured in triplicate, showed consistent initial boiling temperatures, and temperatures in the late stages of the distillation (approaching $75 \%$ DVF) were reproducible within $1.5^{\circ} \mathrm{C}$. The maximum difference between the curves was $4.8{ }^{\circ} \mathrm{C}$, at $30 \%$ DVF (Figure S1). These minor differences are attributable to the physical changes made to the classical ADC to create the ADCR: the substitution of the threeway valve with polytetrafluoroethylene plug for an ordinary distillation head.

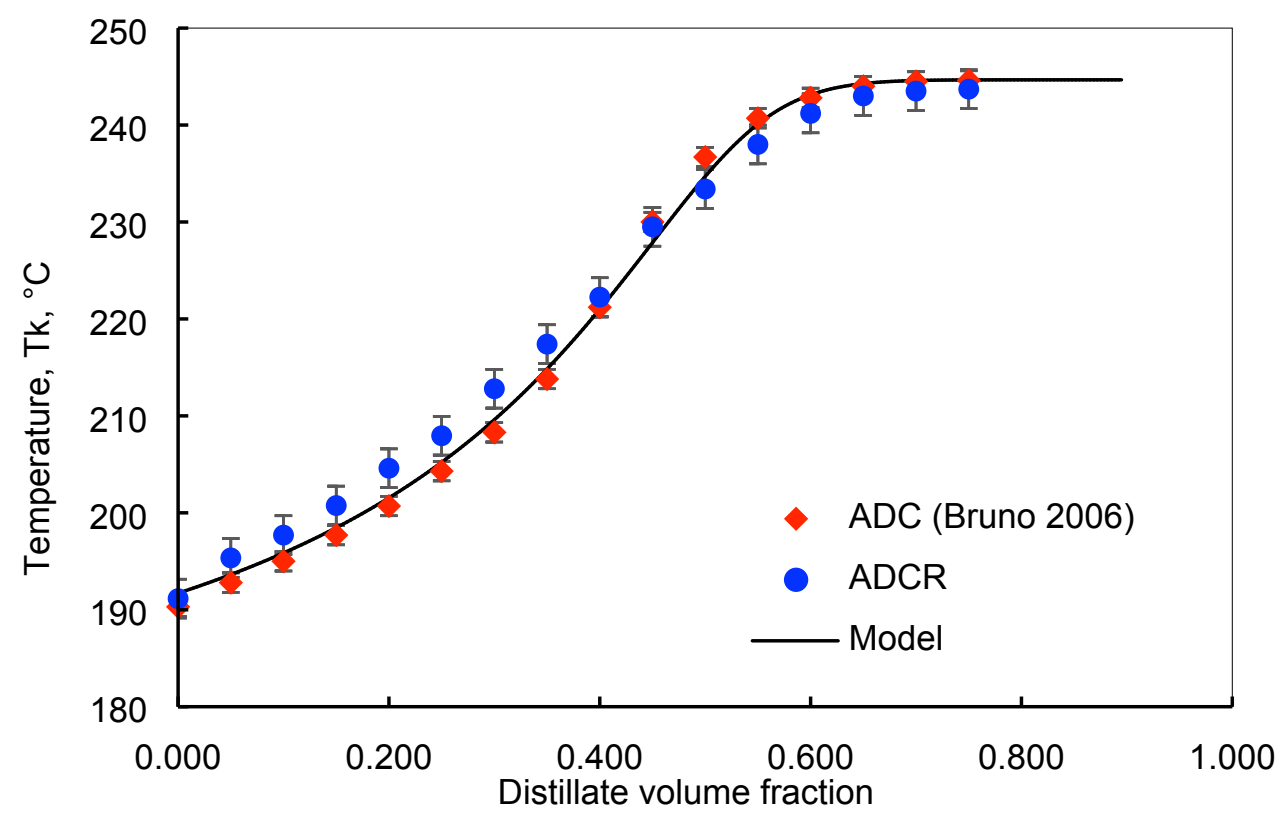

Figure S1. Distillation curve measurements of a 50/50 C10/C14 (mol/mol) mixture, conducted with the modified ADCR apparatus and compared to the original ADC data from 2006. All temperatures were measured at atmospheric pressure in Boulder, $\mathrm{CO}$, about $83 \mathrm{kPa}$, and were not 
corrected. The uncertainty of the ADCR kettle temperatures $\left(\mathrm{T}_{\mathrm{k}}\right)$ is $2{ }^{\circ} \mathrm{C}$. The uncertainty of $\mathrm{T}_{\mathrm{k}}$ in Bruno 2006 is $1^{\circ} \mathrm{C}$. In some cases, error bars are smaller than the markers.

The same comparisons between ADCR measurements and previously published ADC data were made for the Huber-Bruno surrogate (Figure S2). ${ }^{2}$ Agreement among kettle temperatures for this mixture was good. The measured initial boiling temperatures (IBTs) were identical. Measured temperatures were slightly higher in the ADCR measurements across the distillation curve. The maximum difference between the measurements was $5.2{ }^{\circ} \mathrm{C}$. These distillation curves were used to improve the binary interaction parameters for $1,2,4-$ trimethylbenzene (TMB) with each of the alkanes.

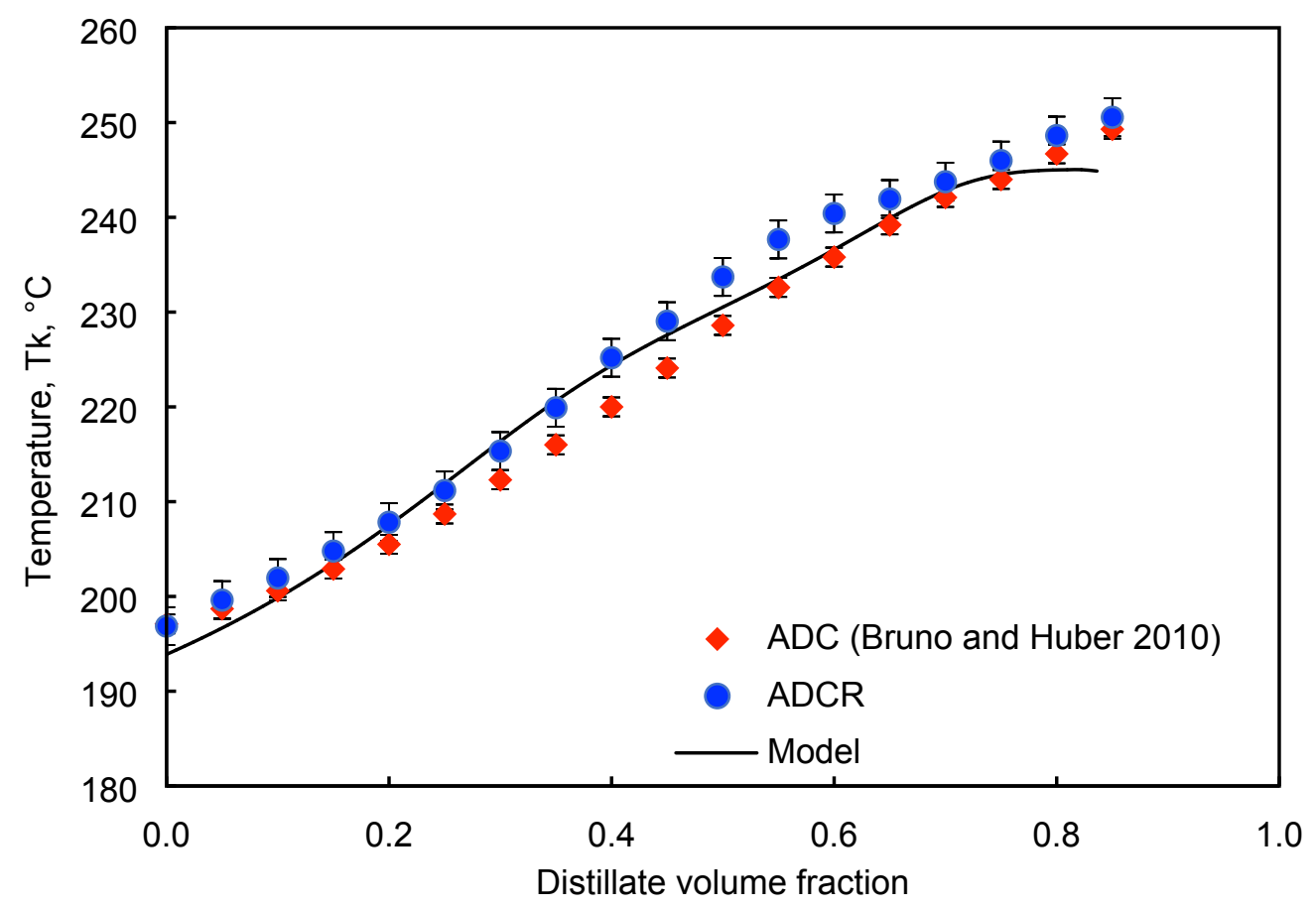

Figure S2. Comparison of kettle temperatures from a distillation of HB surrogate conducted with the modified ADCR apparatus to the original ADC data from 2010. ${ }^{2}$ These curves were measured at atmospheric pressure in Boulder, $\mathrm{CO}$, about $83 \mathrm{kPa}$. The uncertainty of the ADCR kettle temperatures $\left(T_{\mathrm{k}}\right)$ is $2{ }^{\circ} \mathrm{C}$. The uncertainty of $T_{\mathrm{k}}$ in Bruno and Huber 2010 is $1{ }^{\circ} \mathrm{C}$. In some cases, error bars are smaller than the markers. 
From these experiments, it was concluded that distillation curves measured using the ADCR were consistent with previous ADC measurements. Head temperatures were also comparable, and both $T_{\mathrm{k}}$ and $T_{\mathrm{h}}$ were recorded throughout the remaining experiments. 
Table S1. Measured values, their corresponding uncertainties, and modeled predictions of the binary mixture VLE by mass fraction are presented. $x$ represents liquid composition and $y$ represents vapor composition. Uncertainties $(U)$ were calculated with a coverage factor $k=2$.

\begin{tabular}{|c|c|c|c|c|c|c|c|c|c|}
\hline & & \multicolumn{4}{|c|}{$x \mathrm{C}_{14}$ (liquid) } & \multicolumn{4}{|c|}{$y \mathrm{C}_{14}$ (vapor) } \\
\hline$T_{\mathrm{k}},{ }^{\circ} \mathrm{C}$ & $P, \mathrm{kPa}$ & Measured & $U$ & Model & Model-Meas. & Measured & $U$ & Model & Model-Meas. \\
\hline 171.2 & 83.25 & 0.124 & 0.045 & 0.175 & 0.051 & 0.016 & 0.006 & 0.018 & 0.002 \\
\hline 178.7 & 83.05 & 0.335 & 0.048 & 0.375 & 0.040 & 0.035 & 0.008 & 0.048 & 0.013 \\
\hline 182.0 & 83.30 & 0.410 & 0.034 & 0.445 & 0.036 & 0.056 & 0.011 & 0.063 & 0.007 \\
\hline 189.6 & 83.06 & 0.547 & 0.038 & 0.577 & 0.030 & 0.083 & 0.024 & 0.100 & 0.016 \\
\hline 197.7 & 83.16 & 0.638 & 0.054 & 0.682 & 0.044 & 0.134 & 0.021 & 0.145 & 0.011 \\
\hline 205.0 & 83.26 & 0.729 & 0.038 & 0.757 & 0.028 & 0.218 & 0.038 & 0.192 & -0.027 \\
\hline 211.5 & 82.97 & 0.785 & 0.038 & 0.814 & 0.029 & 0.236 & 0.027 & 0.240 & 0.004 \\
\hline 216.3 & 83.47 & 0.830 & 0.031 & 0.852 & 0.023 & 0.270 & 0.055 & 0.282 & 0.012 \\
\hline 223.4 & 83.36 & 0.870 & 0.036 & 0.896 & 0.027 & 0.334 & 0.082 & 0.348 & 0.014 \\
\hline 230.9 & 82.90 & 0.926 & 0.031 & 0.939 & 0.013 & 0.442 & 0.116 & 0.455 & 0.013 \\
\hline 236.1 & 83.43 & 0.944 & 0.035 & 0.964 & 0.019 & 0.581 & 0.074 & 0.545 & -0.036 \\
\hline 241.2 & 83.28 & 0.974 & 0.037 & 0.987 & 0.012 & 0.695 & 0.229 & 0.699 & 0.004 \\
\hline \multirow[t]{2}{*}{244.5} & 83.63 & 0.994 & 0.001 & 0.999 & 0.005 & 0.898 & 0.063 & 0.962 & 0.064 \\
\hline & & \multicolumn{4}{|c|}{$x \mathrm{C}_{10}$ (liquid) } & \multicolumn{4}{|c|}{$y C_{10}$ (vapor) } \\
\hline$T_{\mathrm{k}},{ }^{\circ} \mathrm{C}$ & $P, \mathrm{kPa}$ & Measured & $U$ & Model & Model-Meas. & Measured & $U$ & Model & Model-Meas. \\
\hline 171.2 & 83.25 & 0.876 & 0.061 & 0.825 & -0.051 & 0.984 & 0.029 & 0.982 & -0.002 \\
\hline 178.7 & 83.05 & 0.665 & 0.057 & 0.625 & -0.040 & 0.965 & 0.008 & 0.952 & -0.013 \\
\hline 182.0 & 83.30 & 0.590 & 0.040 & 0.555 & -0.036 & 0.944 & 0.745 & 0.937 & -0.007 \\
\hline 189.6 & 83.06 & 0.453 & 0.039 & 0.423 & -0.030 & 0.917 & 0.024 & 0.900 & -0.016 \\
\hline 197.7 & 83.16 & 0.362 & 0.049 & 0.318 & -0.044 & 0.866 & 0.021 & 0.855 & -0.011 \\
\hline 205.0 & 83.26 & 0.271 & 0.029 & 0.243 & -0.028 & 0.782 & 0.038 & 0.808 & 0.027 \\
\hline 211.5 & 82.97 & 0.215 & 0.026 & 0.186 & -0.029 & 0.764 & 0.027 & 0.760 & -0.004 \\
\hline 216.3 & 83.47 & 0.170 & 0.017 & 0.148 & -0.023 & 0.730 & 0.055 & 0.718 & -0.012 \\
\hline 223.4 & 83.36 & 0.130 & 0.019 & 0.104 & -0.027 & 0.666 & 0.082 & 0.652 & -0.014 \\
\hline 230.9 & 82.90 & 0.074 & 0.012 & 0.061 & -0.013 & 0.558 & 0.116 & 0.545 & -0.013 \\
\hline 236.1 & 83.43 & 0.056 & 0.014 & 0.036 & -0.019 & 0.419 & 0.074 & 0.455 & 0.036 \\
\hline 241.2 & 83.28 & 0.026 & 0.014 & 0.013 & -0.012 & 0.305 & 0.229 & 0.301 & -0.004 \\
\hline 244.5 & 83.63 & 0.006 & 0.001 & 0.001 & 0.005 & 0.102 & 0.063 & 0.038 & 0.064 \\
\hline
\end{tabular}


Table S2. Measured values, their corresponding uncertainties, and model predictions of the Huber-Bruno surrogate VLE by mass fraction are presented. $x$ represents liquid composition and $y$ represents vapor composition. Uncertainties $(U)$ were calculated with a coverage factor $k=2$.

\begin{tabular}{|c|c|c|c|c|c|c|c|c|c|}
\hline \multirow[b]{2}{*}{$T_{\mathrm{k}},{ }^{\circ} \mathrm{C}$} & \multirow[b]{2}{*}{$P, \mathrm{kPa}$} & \multicolumn{4}{|c|}{$x$ TMB } & \multicolumn{4}{|c|}{$y$ TMB } \\
\hline & & Measured & $\boldsymbol{U}$ & Model & Model-Meas & Measured & $U$ & Model & Model-Meas \\
\hline 197.1 & 82.91 & 0.250 & 0.020 & 0.282 & 0.032 & 0.708 & 0.036 & 0.797 & 0.089 \\
\hline 207.0 & 82.86 & 0.150 & 0.012 & 0.166 & 0.017 & 0.676 & 0.018 & 0.675 & -0.002 \\
\hline 217.0 & 82.55 & 0.083 & 0.006 & 0.067 & -0.016 & 0.542 & 0.042 & 0.478 & -0.064 \\
\hline 227.0 & 82.86 & 0.026 & 0.005 & 0.011 & -0.014 & 0.272 & 0.011 & 0.123 & -0.149 \\
\hline \multirow[t]{2}{*}{237.0} & 82.92 & 0.001 & 0.001 & 0.000 & -0.001 & 0.020 & 0.005 & 0.003 & -0.017 \\
\hline & & \multicolumn{4}{|c|}{$x \mathrm{C}_{12}$} & \multicolumn{4}{|c|}{$y C_{12}$} \\
\hline$T_{\mathrm{k}},{ }^{\circ} \mathrm{C}$ & $P, \mathbf{k P a}$ & Measured & $\boldsymbol{U}$ & Model & Model-Meas & Measured & $\boldsymbol{U}$ & Model & Model-Meas \\
\hline 197.1 & 82.91 & 0.324 & 0.012 & 0.332 & 0.009 & 0.209 & 0.012 & 0.168 & -0.041 \\
\hline 207.0 & 82.86 & 0.347 & 0.009 & 0.361 & 0.014 & 0.240 & 0.010 & 0.263 & 0.023 \\
\hline 217.0 & 82.55 & 0.350 & 0.014 & 0.359 & 0.009 & 0.330 & 0.025 & 0.407 & 0.076 \\
\hline 227.0 & 82.86 & 0.328 & 0.014 & 0.294 & -0.035 & 0.486 & 0.032 & 0.609 & 0.123 \\
\hline \multirow[t]{2}{*}{237.0} & 82.92 & 0.221 & 0.016 & 0.126 & -0.095 & 0.531 & 0.037 & 0.471 & -0.060 \\
\hline & & \multicolumn{4}{|c|}{$x \mathrm{C}_{14}$} & \multicolumn{4}{|c|}{$y \mathrm{C}_{14}$} \\
\hline$T_{\mathrm{k}},{ }^{\circ} \mathrm{C}$ & $P, \mathrm{kPa}$ & Measured & $U$ & Model & Model-Meas & Measured & $U$ & Model & Model-Meas \\
\hline 197.1 & 82.91 & 0.427 & 0.019 & 0.385 & -0.041 & 0.083 & 0.011 & 0.035 & -0.047 \\
\hline 207.0 & 82.86 & 0.503 & 0.019 & 0.473 & -0.031 & 0.083 & 0.003 & 0.063 & -0.021 \\
\hline 217.0 & 82.55 & 0.567 & 0.015 & 0.574 & 0.007 & 0.128 & 0.022 & 0.116 & -0.012 \\
\hline 227.0 & 82.86 & 0.646 & 0.018 & 0.695 & 0.049 & 0.241 & 0.023 & 0.268 & 0.027 \\
\hline 237.0 & 82.92 & 0.778 & 0.034 & 0.874 & 0.096 & 0.449 & 0.043 & 0.526 & 0.077 \\
\hline
\end{tabular}

\section{Sources of error in liquid composition measurements for the decane/tetradecane binary}

mixture. Although the uncertainty in vapor composition is greater than the uncertainty in liquid composition, the model and measurements of the liquid do not agree as well as they do for vapor composition. The pattern of disagreement between the liquid composition measurements and model predictions along the bubble curve suggests a possibly systematic error. Although in each case the error bars on the measurements intersect the modeled curve, the model consistently predicts a greater concentration of $\mathrm{C} 14$ in the liquid than was measured at each temperature. If 
this error is truly systematic, either the model or the measurements must be wrong in some manner which pushes the two apart (or both are slightly wrong in opposite directions). The model could be inaccurate due to problems with the individual component equations of state or the binary interaction parameters in the mixture model. The assumptions made in modeling the equilibrium stage in the apparatus could be flawed, although including a stage in the model mostly affects the vapor composition and causes minimal change in the liquid composition compared to a model with no stage. If the model is reliable and instead there is some systematic error in the measurement of kettle composition that caused an undercalculation of $\mathrm{C} 14$, its source has not been identified. The sources of systematic error that have been considered would cause overcalculation of $\mathrm{C} 14$, moving the measurements down below the modeled bubble curve instead of above it.

Several sources of error were considered. First, before collecting the liquid sample, the magnetic stir bar is used to thoroughly mix the fluid remaining in the kettle. Any stratification that may occur because of differences in density were therefore eliminated. Next, the effect of any vapor condensate holdup was considered (in the reflux condenser, inside the channel of the reflux junction, or coating the walls of the glassware); however, the effect, if it were significant, of such material being lost from the kettle would be an overcalculation of the C14 concentration. This held-up condensed vapor would be enriched in C10, the more volatile component, causing the remaining kettle liquid to contain more C14, not less. Any effect of vapor forming in equilibrium above the kettle liquid (in the headspace) would also cause an offset in the wrong direction. Finally, the error could be occurring in the temperature measurement due to a lag in thermocouple or readout response, but such a lag would have been reproduced in the initial comparison of the distillation curve with classical ADC measurements. This would shift the 
measurements to lower temperatures and again, below the model prediction - not above it, as is the case. Error in temperatures sensed by the thermocouples is prevented by regular calibration using an indium cell according to NIST protocol. Therefore, the minor disagreement between the modeled and measured bubble curves could not be definitively explained. The magnitude of the disagreement is small—smaller than the uncertainty in the measurements - but the source of a possibly systematic error should be investigated.

\section{References}

1. Bruno, T. J., Improvements in the measurement of distillation curves. 1. A compositionexplicit approach. Ind Eng Chem Res 2006, 45(12): 4371-4380.

2. Bruno, T. J.; Huber, M.L., Evaluation of the physicochemical authenticity of aviation kerosene surrogate mixtures. Part 2: Analysis and prediction of thermophysical properties. Energy Fuels 2010, 24(8): 4277-4284. 